\title{
Fatty acid composition of wild edible mushrooms species: A comparative study
}

\author{
Bárbara Ribeiro $^{\text {a }}$, Paula Guedes de Pinho ${ }^{\mathrm{a}, *}$, Paula B. Andrade ${ }^{a}$, Paula Baptista ${ }^{\mathrm{b}}$, Patrícia Valentão ${ }^{\mathrm{a}, *}$ \\ a REQUIMTE/Serviço de Farmacognosia, Faculdade de Farmácia da Universidade do Porto, R. Aníbal Cunha 164, 4050-047 Porto, Portugal \\ b CIMO/Escola Superior Agrária, Instituto Politécnico de Bragança, Campus de Sta Apolónia, Apartado 1172, 5301-855 Bragança, Portugal
}

\section{A R T I C L E I N F O}

\section{Article history:}

Received 5 April 2009

Accepted 12 April 2009

Available online 24 April 2009

\section{Keywords:}

Wild edible mushrooms

Fatty acids

Comparative analysis

\begin{abstract}
A B S T R A C T
A comparative study was developed on the total fatty acids composition of twelve wild edible mushroom species (Suillus bellini, Suillus luteus, Suillus granulatus, Hygrophorus agathosmus, Amanita rubescens, Russula cyanoxantha, Boletus edulis, Tricholoma equestre, Fistulina hepatica, Cantharellus cibarius, Amanita caesarea and Hydnum rufescens). In order to define qualitative and quantitative profiles, combined fatty acids were hydrolyzed with potassium hydroxide/methanol and all free compounds were derived to their methyl ester forms with methanolic boron trifluoride, followed by analysis by GC-MS. Thirty fatty acids were determined. As far as we know, the fatty acid profiles of A. caesarea and H. rufescens are described for the first time. As for the remaining species, a high number of new compounds were identified, which much improved the knowledge about their fatty acids profiles. In general, oleic, linoleic, palmitic and stearic acids were present in highest contents. Polyunsaturated and monounsaturated fatty acids, valuable healthy compounds for humans, predominated over saturated fatty acids for all the studied mushroom species. $R$. cyanoxantha presented the highest fatty acids amounts, while $B$. edulis was the poorest species. By Agglomerative Hierarchic Cluster Analysis the studied species were gathered in 5 groups, based in their fatty acid patterns. (c) 2009 Elsevier B.V. All rights reserved.
\end{abstract}

\section{Introduction}

Wild edible mushrooms are widely consumed in many countries. Their culinary and commercial value is mainly due to their organoleptic properties, such as aroma and flavour [1], and also to their riches in carbohydrates, fibres [2], vitamins and minerals, additionally containing high proportions of unsaturated fatty acids [3]. The high protein and low fat/energy contents of wild edible mushrooms make them an excellent food for use in low caloric diets [4].

Lipids display an important role in human body, acting like hormones or their precursors, helping the digestion process, and constituting a source of metabolic energy. They also work as structural and functional components of biomembranes, as constituents of myelin sheath and as thermal insulators [5].

Fatty acids are the basic building blocks of most lipids. Polyunsaturated fatty acids from omega- 6 and omega- 3 families have intense biological properties in low concentrations [6] and are the biosynthetic precursors of the eicosanoids (i.e. prostaglandins). These are signalling molecules with complex control over many body systems, having effects on cardiovascular diseases, triglycerides levels, blood pressure and arthritis [7].

Because of its weather conditions and flora diversity, Trás-osMontes (northeastern Portugal) is one of the European regions with wide mycological patrimony in wild mushrooms of considerable

\footnotetext{
* Corresponding authors. Tel.: +351 222078935; fax: +351 222003977.

E-mail addresses: pguedes@ff.up.pt (P. Guedes de Pinho), valentao@ff.up.pt (P. Valentão)
}

gastronomic relevance. Moreover, the harvest of those species constitutes a way of subsistence for the local residents, playing an important role in the regional and national commerce.

In the present work twelve wild edible mushrooms species collected in this region were studied: Suillus bellini, Suillus luteus, Suillus granulatus, Hygrophorus agathosmus, Amanita rubescens, Russula cyanoxantha, Boletus edulis, Tricholoma equestre, Fistulina hepatica, Cantharellus cibarius, Amanita caesarea and Hydnum rufescens.

Previous studies described the presence of some fatty acids in S. luteus and A. rubescens [8], in B. edulis and C. cibarius [9] and in $F$. hepatica [10]. In a previous work concerning the pattern volatiles of wild edible mushrooms, seven fatty acids were identified (butyric, caproic, myristoleic, palmitoleic, stearic, linoleic and oleic acids) [1]. Some of them were found in S. granulatus, S. bellini, R. cyanoxantha, $H$. agathosmus and T. equestre. As far as we know, nothing has been described for $A$. caesarea and $H$. rufescens fatty acids profiles.

Usually, the analysis of fatty acids from lipids is performed by gaschromatography (GC) and involves a pre-derivatization of the combined fatty acids into less polar and more volatile compounds, so that they can be eluted at reasonable temperatures, without thermal decomposition or molecular rearrangement along the GC column. The most common procedure consists in the hydrolysis and methylation of the combined fatty acids to form their respective methyl esters (FAMEs) in the presence of a catalytic acid, such as boron trifluoride $\left(\mathrm{BF}_{3}\right)[8,11]$.

In the sequence of the chemical characterization that we have been developing on the mushroom species referred above [1,12-15], the study of their fatty acids composition was conducted, in order to 
define or improve the knowledge of their profiles and, ultimately, to compare the species.

\section{Materials and methods}

\subsection{Standards and reagents}

Caproic, caprylic, capric, undecanoic, lauric, tridecanoic, myristic, myristoleic, pentadecanoic, palmitic, palmitoleic, heptadecanoic, cis-10heptadecenoic, stearic, oleic, linoleic, $\gamma$-linolenic, cis-11-eicosenoic, $\alpha$ linolenic, arachidic, cis-11,14-eicosadienoic, cis-8,11,14-eicosatrienoic, arachidonic, cis-5,8,11,14,17-eicosapentaenoic, heneicosanoic, behenic, erucic, cis-4,7,10,13,16,19-docosahexaenoic, tricosanoic, lignoceric and nervonic acid methyl esters, and boron trifluoride $\left(\mathrm{BF}_{3}\right) 10 \%$ methanolic solution were purchased from Supelco (Bellefonte, PA, USA). Chloroform, methanol, anhydrous sodium sulphate and isooctane were from Panreac Quimica SA (Barcelona, Spain). Potassium hydroxide was obtained from Pronalab (Lisboa, Portugal). Methyl jasmonate (Internal Standard) was from by SAFC (St. Louis, USA) and dichloromethane from Merck (Darmsatdt, Germany).

\subsection{Samples}

Samples of wild edible mushroom species were collected in Trásos-Montes region (northeastern of Portugal), and their characterization is provided in Table 1. After harvesting, the mushrooms were immediately transferred to the laboratory. Taxonomic identification followed that of several authors [16-21] and representative voucher specimens were deposited at the herbarium of Escola Superior Agrária of Instituto Politécnico de Bragança. Samples were dehydrated in a ventilated oven at $30^{\circ} \mathrm{C}$, for 7 days. All materials were kept in the dark, in hermetically sealed bags, and powdered $(910 \mu \mathrm{m})$ before analysis.

\subsection{Extracts preparation}

For the extracts' preparation and fatty acids derivatization, we used the methods of León-Guzmán et al. [8] with minor modifications: ca. $1.5 \mathrm{~g}$ of the powdered mushroom plus $200 \mu \mathrm{L}$ of methyl jasmonate $1 \mathrm{~g} / \mathrm{L}$ methanolic solution (internal standard) were mixed with chloroform: methanol $(2: 1)(5 \times 150 \mathrm{~mL})$ with magnetic stirring at $500 \mathrm{rpm}$, for $10 \mathrm{~min}$, at $40{ }^{\circ} \mathrm{C}$. The resulting mixtures were concentrated to dryness under reduced pressure $\left(40^{\circ} \mathrm{C}\right)$ and redissolved in $5 \mathrm{~mL}$ of methanol.

\subsection{Derivatization}

The lipids in the extract were hydrolyzed with $1 \mathrm{~mL}$ of $\mathrm{KOH}$ methanolic solution $(11 \mathrm{~g} / \mathrm{L})$, at $90{ }^{\circ} \mathrm{C}$ for $10 \mathrm{~min}$. The free fatty acids originally present and those resulting from the alkaline hydrolysis

Table 1

Characterization of mushroom samples.

\begin{tabular}{|c|c|c|c|c|}
\hline Sample & Species & Origin & Orchard & $\begin{array}{l}\text { Date of } \\
\text { collection }\end{array}$ \\
\hline 1 & Suillus bellini & Bragança & Pinus pinaster & November 2004 \\
\hline 2 & Suillus luteus & Vinhais & $\begin{array}{l}\text { Pinus pinaster }+ \\
\text { Castanea sativa }\end{array}$ & November 2005 \\
\hline 3 & Suillus granulatus & Bragança & Pinus pinaster & November 2005 \\
\hline 4 & Hygrophorus agathosmus & Bragança & Pinus pinaster & November 2004 \\
\hline 5 & Amanita rubescens & Bragança & Quercus pyrenaica & May 2006 \\
\hline 6 & Russula cyanoxantha & Bragança & Quercus rotundifolia & November 2006 \\
\hline 7 & Boletus edulis & Bragança & Castanea sativa & June 2005 \\
\hline 8 & Tricholoma equestre & $\begin{array}{l}\text { Carrazeda de } \\
\text { Ansiães }\end{array}$ & Pinus pinaster & November 2005 \\
\hline 9 & Fistulina hepatica & Bragança & Castanea sativa & October 2005 \\
\hline 10 & Cantharellus cibarius & Grandais & Castanea sativa & November 2006 \\
\hline 11 & Amanita caesarea & Grandais & Castanea sativa & October 2006 \\
\hline 12 & Hydnum rufescens & Grandais & Castanea sativa & November 2006 \\
\hline
\end{tabular}

were derivatized to their methyl ester forms with $1 \mathrm{~mL}$ of BF3 methanolic solution (10\%), at $90{ }^{\circ} \mathrm{C}$ for $10 \mathrm{~min}$. The methyl esters derivatives were purified with $2 \times 6 \mathrm{~mL}$ of isooctane and anhydrous sodium sulphate was added to assure the total absence of water. The resulting extract was evaporated to dryness under a stream of nitrogen and redissolved in $400 \mu \mathrm{L}$ of isooctane. All the assays were performed in triplicate for each mushroom species.

\subsection{Calibration curve}

A methyl ester mixture of saturated fatty acids (SFA) (caproic, caprylic, capric, undecanoic, lauric, tridecanoic, myristic, pentadecanoic, palmitic, heptadecanoic, stearic, arachidic, heneicosanoic, behenic, tricosanoic and lignoceric acids), monounsaturated fatty acids (MUFA) (myristoleic, palmitoleic, cis-10-heptadecenoic, oleic, cis-11-eicosenoic, erucic and nervonic acids) and polyunsaturated fatty acids (PUFA) (linoleic, $\gamma$-linolenic, $\alpha$-linolenic, cis-11,14-eicosadienoic, cis-8,11,14eicosatrienoic, arachidonic, cis-5,8,11,14,17-eicosapentaenoic and cis$4,7,10,13,16,19$-docosahexaenoic acids), in a concentration series of 4.00 , $2.00,1.00,0.50$ and $0.25 \mathrm{mg} / \mathrm{L}$, was prepared in isooctane.

\subsection{Gas chromatography-mass spectrometry analysis}

Standard mixture/sample extracts $(1 \mu \mathrm{L})$ were analyzed using a Varian CP-3800 gas chromatograph (USA) equipped with a VARIAN Saturn 4000 mass selective detector (USA) and a Saturn GC/MS workstation software version 6.8. A VF- $5 \mathrm{~ms}(30 \mathrm{~m} \times 0.25 \mathrm{~mm} \times 0.25 \mu \mathrm{m})$ column (VARIAN) was used. The injector port was heated to $250^{\circ} \mathrm{C}$. Injections were performed in split mode, with a ratio of 1/40. The carrier gas was Helium C-60 (Gasin, Portugal), at a constant flow of $1 \mathrm{~mL} / \mathrm{min}$. The oven temperature was set at $40{ }^{\circ} \mathrm{C}$ for $1 \mathrm{~min}$, then increasing $5^{\circ} \mathrm{C} / \mathrm{min}$ to $250^{\circ} \mathrm{C}, 3^{\circ} \mathrm{C} / \mathrm{min}$ to $300^{\circ} \mathrm{C}$ and held for $15 \mathrm{~min}$. All mass spectra were acquired in electron impact (EI) mode. Ionization was maintained off during the first $4 \mathrm{~min}$, to avoid solvent overloading. The Ion Trap detector was set as follows: transfer line, manifold and trap temperatures were respectively 280,50 and $180^{\circ} \mathrm{C}$. The mass ranged from 50 to $600 \mathrm{~m} / z$, with a scan rate of $6 \mathrm{scan} / \mathrm{s}$. The emission current was $50 \mu \mathrm{A}$, and the electron multiplier was set in relative mode to auto tune procedure. The maximum ionization time was $25,000 \mu$ s, with an ionization storage level of $35 \mathrm{~m} / \mathrm{z}$. The injection volume for liquid extracts was $1 \mu \mathrm{L}$ and the analysis was performed in Full Scan mode.

Identification of compounds was achieved by comparisons of their mass spectra with those from pure standards injected under the same conditions, and from NIST 05 MS Library Database. The amount of FAMEs present in the extract samples was achieved from the calibration curve of the respective FAME standards. The FAMEs values were then converted into their respective fatty acid contents.

\subsection{Statistical analysis}

Agglomerative hierarchic cluster analysis (HCA) was carried out using XLSTAT 2007.5 software. The dendogram method shows correlations through clusters diagrams.

\section{Results and discussion}

The study of the fatty acids composition of $S$. bellini, S. luteus, S. granulatus, H. agathosmus, A. rubescens, R. cyanoxantha, B. edulis, T. equestre, F. hepatica, $C$. cibarius, A. caesarea and $H$. rufescens species revealed the presence of caproic, caprylic, capric, undecanoic, lauric, tridecanoic, myristoleic, myristic, pentadecanoic, palmitoleic, palmitic, cis-10-heptadecenoic, heptadecanoic, $\gamma$-linolenic, linoleic, oleic, stearic, arachidonic, cis-5,8,11,14,17-eicosapentaenoic, cis-8,11,14-eicosatrienoic, cis-11,14-eicosadienoic, cis-11-eicosaenoic acid, $\alpha$-linolenic, arachidic, heneicosanoic, erucic, behenic, tricosanoic, nervonic and lignoceric acids (Table 2, Fig. 1). The fatty acids contents in the analyzed samples ranged from ca. 30 to $3175 \mathrm{mg} / \mathrm{kg}$ (Table 2). $R$. cyanoxantha, followed by 
Table 2

Free fatty acids composition of mushroom species ( $\mathrm{mg} / \mathrm{kg}$ dry matter). ${ }^{\mathrm{a}}$

\begin{tabular}{|c|c|c|c|c|c|c|c|c|c|c|c|c|}
\hline \multirow[t]{2}{*}{ Fatty acid } & \multicolumn{12}{|l|}{ Samples } \\
\hline & S. bellini & S. luteus & S. granulatus & H. agathosmus & A. rubescens & R. cyanoxantha & B. edulis & T. equestre & F. hepatica & C. cibarius & A. caesarea & H. rufescens \\
\hline$\overline{\mathrm{C} 6: 0}$ & nd & $\begin{array}{l}0.003 \\
(0.000)\end{array}$ & nd & $\begin{array}{l}0.021 \\
(0.000)\end{array}$ & nd & $\begin{array}{l}0.029 \\
(0.001)\end{array}$ & nd & $\begin{array}{l}0.009 \\
(0.001)\end{array}$ & nd & nd & $\begin{array}{l}0.015 \\
(0.000)\end{array}$ & $\begin{array}{l}0.068 \\
(0.001)\end{array}$ \\
\hline C8:0 & $\begin{array}{l}0.892 \\
(0.070)\end{array}$ & $\begin{array}{l}0.015 \\
(0.001)\end{array}$ & $\begin{array}{l}0.014 \\
(0.000)\end{array}$ & $\begin{array}{l}0.040 \\
(0.004)\end{array}$ & nd & $\begin{array}{l}0.045 \\
(0.002)\end{array}$ & nd & $\begin{array}{l}0.049 \\
(0.002)\end{array}$ & $\begin{array}{l}0.007 \\
(0.000)\end{array}$ & nd & $\begin{array}{l}0.027 \\
(0.001)\end{array}$ & $\begin{array}{l}0.089 \\
(0.001)\end{array}$ \\
\hline C10:0 & $\begin{array}{l}1.688 \\
(0.101)\end{array}$ & $\begin{array}{l}0.013 \\
(0.001)\end{array}$ & $\begin{array}{l}0.039 \\
(0.001)\end{array}$ & $\begin{array}{l}0.013 \\
(0.001)\end{array}$ & $\begin{array}{l}0.005 \\
(0.000)\end{array}$ & $\begin{array}{l}0.244 \\
(0.013)\end{array}$ & $\begin{array}{l}0.008 \\
(0.002)\end{array}$ & $\begin{array}{l}0.026 \\
(0.002)\end{array}$ & $\begin{array}{l}0.004 \\
(0.001)\end{array}$ & nd & $\begin{array}{l}0.010 \\
(0.001)\end{array}$ & nd \\
\hline C11:0 & $\begin{array}{l}0.165 \\
(0.007)\end{array}$ & $\begin{array}{l}0.001 \\
(0.000)\end{array}$ & $\begin{array}{l}0.006 \\
(0.000)\end{array}$ & $\begin{array}{l}0.014 \\
(0.000)\end{array}$ & nd & nd & nd & nd & nd & nd & nd & nd \\
\hline $\mathrm{C} 12: 0$ & $\begin{array}{l}3.605 \\
(0.125)\end{array}$ & $\begin{array}{l}0.059 \\
(0.002)\end{array}$ & $\begin{array}{l}0.143 \\
(0.012)\end{array}$ & $\begin{array}{l}0.170 \\
(0.006)\end{array}$ & $\begin{array}{l}0.128 \\
(0.005)\end{array}$ & $\begin{array}{l}0.281 \\
(0.007)\end{array}$ & $\begin{array}{l}0.027 \\
(0.005)\end{array}$ & $\begin{array}{l}0.019 \\
(0.000)\end{array}$ & $\begin{array}{l}0.016 \\
(0.001)\end{array}$ & $\begin{array}{l}0.055 \\
(0.002)\end{array}$ & $\begin{array}{l}0.041 \\
(0.002)\end{array}$ & $\begin{array}{l}0.029 \\
(0.002)\end{array}$ \\
\hline C13:0 & $\begin{array}{l}3.709 \\
(0.150)\end{array}$ & $\begin{array}{l}0.025 \\
(0.001)\end{array}$ & $\begin{array}{l}0.043 \\
(0.003)\end{array}$ & $\begin{array}{l}1.672 \\
(0.040)\end{array}$ & $\begin{array}{l}0.026 \\
(0.001)\end{array}$ & $\begin{array}{l}0.030 \\
(0.002)\end{array}$ & nd & nd & $\begin{array}{l}0.005 \\
(0.000)\end{array}$ & nd & $\begin{array}{l}0.009 \\
(0.000)\end{array}$ & $\begin{array}{l}0.009 \\
(0.000)\end{array}$ \\
\hline C14:0 & $\begin{array}{l}18.280 \\
(1.201)\end{array}$ & $\begin{array}{l}0.520 \\
(0.027)\end{array}$ & $\begin{array}{l}1.694 \\
(0.173)\end{array}$ & $\begin{array}{l}0.715 \\
(0.029)\end{array}$ & $\begin{array}{l}1.361 \\
(0.063)\end{array}$ & $\begin{array}{l}0.852 \\
(0.046)\end{array}$ & $\begin{array}{l}0.216 \\
(0.013)\end{array}$ & $\begin{array}{l}0.272 \\
(0.008)\end{array}$ & $\begin{array}{l}0.150 \\
(0.004)\end{array}$ & $\begin{array}{l}2.315 \\
(0.247)\end{array}$ & $\begin{array}{l}0.534 \\
(0.015)\end{array}$ & $\begin{array}{l}0.298 \\
(0.032)\end{array}$ \\
\hline C14:1 & nd & $\begin{array}{l}0.006 \\
(0.000)\end{array}$ & $\begin{array}{l}0.020 \\
(0.001)\end{array}$ & nd & nd & $\begin{array}{l}0.004 \\
(0.001)\end{array}$ & nd & nd & nd & nd & $\begin{array}{l}0.012 \\
(0.000)\end{array}$ & nd \\
\hline C15:0 & $\begin{array}{l}25.298 \\
(1.070)\end{array}$ & $\begin{array}{l}1.462 \\
(0.106)\end{array}$ & $\begin{array}{l}3.307 \\
(0.002)\end{array}$ & $\begin{array}{l}1.229 \\
(0.101)\end{array}$ & $\begin{array}{l}2.421 \\
(0.182)\end{array}$ & $\begin{array}{l}1.330 \\
(0.055)\end{array}$ & $\begin{array}{l}0.349 \\
(0.003)\end{array}$ & $\begin{array}{l}1.259 \\
(0.072)\end{array}$ & $\begin{array}{l}0.484 \\
(0.012)\end{array}$ & $\begin{array}{l}0.523 \\
(0.048)\end{array}$ & $\begin{array}{l}0.656 \\
(0.033)\end{array}$ & $\begin{array}{l}0.750 \\
(0.068)\end{array}$ \\
\hline $\mathrm{C} 16: 0$ & $\begin{array}{l}160.702 \\
(0.893)\end{array}$ & $\begin{array}{l}11.209 \\
(1.030)\end{array}$ & $\begin{array}{l}6.926 \\
(0.073)\end{array}$ & $\begin{array}{l}19.045 \\
(1.047)\end{array}$ & $\begin{array}{l}39.761 \\
(0.000)\end{array}$ & $\begin{array}{l}16.015 \\
(1.407)\end{array}$ & $\begin{array}{l}5.285 \\
(0.120)\end{array}$ & $\begin{array}{l}7.312 \\
(0.302)\end{array}$ & $\begin{array}{l}4.857 \\
(0.100)\end{array}$ & $\begin{array}{l}15.660 \\
(0.119)\end{array}$ & $\begin{array}{l}14.101 \\
(1.106)\end{array}$ & $\begin{array}{l}9.933 \\
(0.200)\end{array}$ \\
\hline C16:1 & $\begin{array}{l}16.117 \\
(1.714)\end{array}$ & $\begin{array}{l}1.780 \\
(0.088)\end{array}$ & $\begin{array}{l}4.307 \\
(0.100)\end{array}$ & $\begin{array}{l}1.010 \\
(0.073)\end{array}$ & $\begin{array}{l}1.955 \\
(0.087)\end{array}$ & $\begin{array}{l}1.920 \\
(0.157)\end{array}$ & $\begin{array}{l}0.675 \\
(0.020)\end{array}$ & $\begin{array}{l}0.270 \\
(0.014)\end{array}$ & $\begin{array}{l}0.060 \\
(0.001)\end{array}$ & $\begin{array}{l}0.324 \\
(0.023)\end{array}$ & $\begin{array}{l}1.796 \\
(0.150)\end{array}$ & $\begin{array}{l}1.862 \\
(0.158)\end{array}$ \\
\hline C17:0 & $\begin{array}{l}3.523 \\
(0.311)\end{array}$ & $\begin{array}{l}0.489 \\
(0.006)\end{array}$ & $\begin{array}{l}1.698 \\
(0.114)\end{array}$ & $\begin{array}{l}0.161 \\
(0.006)\end{array}$ & $\begin{array}{l}2.520 \\
(0.148)\end{array}$ & $\begin{array}{l}0.245 \\
(0.015)\end{array}$ & $\begin{array}{l}0.225 \\
(0.003)\end{array}$ & $\begin{array}{l}0.176 \\
(0.014)\end{array}$ & $\begin{array}{l}0.212 \\
(0.011)\end{array}$ & $\begin{array}{l}0.504 \\
(0.000)\end{array}$ & $\begin{array}{l}0.166 \\
(0.000)\end{array}$ & $\begin{array}{l}0.442 \\
(0.05)\end{array}$ \\
\hline C17:1 & nd & $\begin{array}{l}0.259 \\
(0.003)\end{array}$ & $\begin{array}{l}1.094 \\
(0.021)\end{array}$ & $\begin{array}{l}0.084 \\
(0.005)\end{array}$ & nd & nd & $\begin{array}{l}0.133 \\
(0.004)\end{array}$ & nd & $\begin{array}{l}0.028 \\
(0.002)\end{array}$ & nd & $\begin{array}{l}0.098 \\
(0.006)\end{array}$ & $\begin{array}{l}0.040 \\
(0.001)\end{array}$ \\
\hline C18:0 & $\begin{array}{l}76.787 \\
(4.071)\end{array}$ & $\begin{array}{l}3.107 \\
(0.301)\end{array}$ & $\begin{array}{l}6.690 \\
(0.151)\end{array}$ & $\begin{array}{l}4.481 \\
(0.266)\end{array}$ & $\begin{array}{l}27.844 \\
(1.430)\end{array}$ & $\begin{array}{l}6.941 \\
(0.360)\end{array}$ & $\begin{array}{l}3.084 \\
(0.351)\end{array}$ & $\begin{array}{l}2.787 \\
(0.079)\end{array}$ & $\begin{array}{l}1.782 \\
(0.069)\end{array}$ & $\begin{array}{l}7.017 \\
(0.126)\end{array}$ & $\begin{array}{l}7.111 \\
(0.669)\end{array}$ & $\begin{array}{l}4.066 \\
(0.263)\end{array}$ \\
\hline C18:1 & $\begin{array}{l}191.046 \\
(4.045)\end{array}$ & $\begin{array}{l}1097.004 \\
(12.530)\end{array}$ & $\begin{array}{l}1177.661 \\
(10.159)\end{array}$ & $\begin{array}{l}159.028 \\
(4.284)\end{array}$ & $\begin{array}{l}69.810 \\
(2.813)\end{array}$ & $\begin{array}{l}2165.499 \\
(13.424)\end{array}$ & $\begin{array}{l}11.583 \\
(0.412)\end{array}$ & $\begin{array}{l}244.048 \\
(5.846)\end{array}$ & $\begin{array}{l}8.448 \\
(0.121)\end{array}$ & $\begin{array}{l}148.168 \\
(2.320)\end{array}$ & $\begin{array}{l}1282.709 \\
(13.870)\end{array}$ & $\begin{array}{l}11.798 \\
(0.344)\end{array}$ \\
\hline C18:2 & $\begin{array}{l}124.578 \\
(5.019)\end{array}$ & $\begin{array}{l}650.694 \\
(6.105)\end{array}$ & $\begin{array}{l}663.008 \\
(9.400)\end{array}$ & $\begin{array}{l}76.380 \\
(1.065)\end{array}$ & $\begin{array}{l}19.365 \\
(0.545)\end{array}$ & $\begin{array}{l}976.316 \\
(22.537)\end{array}$ & $\begin{array}{l}7.536 \\
(0.492)\end{array}$ & $\begin{array}{l}203.032 \\
(6.329)\end{array}$ & $\begin{array}{l}18.607 \\
(0.140)\end{array}$ & $\begin{array}{l}654.706 \\
(3.471)\end{array}$ & $\begin{array}{l}310.698 \\
(7.320)\end{array}$ & $\begin{array}{l}7.885 \\
(0.100)\end{array}$ \\
\hline C18:3n6 & nd & nd & nd & nd & nd & $\begin{array}{l}0.022 \\
(0.001)\end{array}$ & nd & $\begin{array}{l}0.019 \\
(0.001)\end{array}$ & nd & nd & nd & nd \\
\hline $\begin{array}{c}\text { C18:3n3+ } \\
\text { C20:1 }\end{array}$ & nd & $\begin{array}{l}0.613 \\
(0.023)\end{array}$ & $\begin{array}{l}0.100 \\
(0.003)\end{array}$ & $\begin{array}{l}1.306 \\
(0.154)\end{array}$ & $\begin{array}{l}0.455 \\
(0.060)\end{array}$ & $\begin{array}{l}0.479 \\
(0.026)\end{array}$ & $\begin{array}{l}0.165 \\
(0.014)\end{array}$ & nd & nd & nd & $\begin{array}{l}0.381 \\
(0.016)\end{array}$ & nd \\
\hline C20:0 & $\begin{array}{l}7.128 \\
(0.104)\end{array}$ & $\begin{array}{l}0.436 \\
(0.022)\end{array}$ & $\begin{array}{l}1.504 \\
(0.047)\end{array}$ & $\begin{array}{l}2.179 \\
(0.175)\end{array}$ & $\begin{array}{l}3.230 \\
(0.141)\end{array}$ & $\begin{array}{l}0.900 \\
(0.044)\end{array}$ & $\begin{array}{l}0.423 \\
(0.056)\end{array}$ & $\begin{array}{l}0.241 \\
(0.013)\end{array}$ & $\begin{array}{l}0.098 \\
(0.005)\end{array}$ & nd & $\begin{array}{l}0.698 \\
(0.038)\end{array}$ & nd \\
\hline $\mathrm{C} 20: 2$ & $\begin{array}{l}0.476 \\
(0.043)\end{array}$ & $\begin{array}{l}0.219 \\
(0.010)\end{array}$ & $\begin{array}{l}0.343 \\
(0.010)\end{array}$ & $\begin{array}{l}0.084 \\
(0.006)\end{array}$ & $\begin{array}{l}0.122 \\
(0.005)\end{array}$ & $\begin{array}{l}0.063 \\
(0.003)\end{array}$ & $\begin{array}{l}0.105 \\
(0.004)\end{array}$ & nd & $\begin{array}{l}0.016 \\
(0.002)\end{array}$ & $\begin{array}{l}0.088 \\
(0.001)\end{array}$ & $\begin{array}{l}0.178 \\
(0.010)\end{array}$ & $\begin{array}{l}0.033 \\
(0.002)\end{array}$ \\
\hline$C 20: 3 n 6$ & $\begin{array}{l}0.476 \\
(0.000)\end{array}$ & $\begin{array}{l}0.015 \\
(0.001)\end{array}$ & $\begin{array}{l}0.021 \\
(0.002)\end{array}$ & $\begin{array}{l}0.023 \\
(0.002)\end{array}$ & $\begin{array}{l}0.015 \\
(0.000)\end{array}$ & nd & $\begin{array}{l}0.004 \\
(0.000)\end{array}$ & nd & nd & $\begin{array}{l}0.088 \\
(0.000)\end{array}$ & $\begin{array}{l}0.013 \\
(0.000)\end{array}$ & nd \\
\hline C20:4n6 & $\begin{array}{l}1.532 \\
(0.051)\end{array}$ & $\begin{array}{l}0.323 \\
(0.008)\end{array}$ & nd & $\begin{array}{l}0.098 \\
(0.003)\end{array}$ & nd & $\begin{array}{l}0.051 \\
(0.001)\end{array}$ & $\begin{array}{l}0.030 \\
(0.002)\end{array}$ & nd & $\begin{array}{l}0.009 \\
(0.000)\end{array}$ & $\begin{array}{l}0.085 \\
(0.006)\end{array}$ & nd & nd \\
\hline$C 20: 5 n 3$ & nd & nd & nd & nd & $\begin{array}{l}0.026 \\
(0.001)\end{array}$ & $\begin{array}{l}0.028 \\
(0.001)\end{array}$ & nd & nd & $\begin{array}{l}0.016 \\
(0.000)\end{array}$ & nd & nd & $\begin{array}{l}0.044 \\
(0.002)\end{array}$ \\
\hline $\mathrm{C} 21: 0$ & $\begin{array}{l}1.705 \\
(0.098)\end{array}$ & $\begin{array}{l}0.094 \\
(0.003)\end{array}$ & $\begin{array}{l}0.435 \\
(0.019)\end{array}$ & $\begin{array}{l}0.031 \\
(0.001)\end{array}$ & $\begin{array}{l}0.065 \\
(0.002)\end{array}$ & $\begin{array}{l}0.031 \\
(0.003)\end{array}$ & $\begin{array}{l}0.015 \\
(0.001)\end{array}$ & $\begin{array}{l}0.049 \\
(0.001)\end{array}$ & $\begin{array}{l}0.010 \\
(0.000)\end{array}$ & nd & $\begin{array}{l}0.041 \\
(0.003)\end{array}$ & nd \\
\hline $\mathrm{C} 22: 0$ & $\begin{array}{l}16.772 \\
(0.890)\end{array}$ & $\begin{array}{l}0.310 \\
(0.003)\end{array}$ & $\begin{array}{l}0.930 \\
(0.069)\end{array}$ & $\begin{array}{l}1.345 \\
(0.101)\end{array}$ & $\begin{array}{l}0.721 \\
(0.038)\end{array}$ & $\begin{array}{l}1.429 \\
(0.074)\end{array}$ & $\begin{array}{l}0.154 \\
(0.012)\end{array}$ & $\begin{array}{l}0.144 \\
(0.006)\end{array}$ & $\begin{array}{l}0.035 \\
(0.000)\end{array}$ & $\begin{array}{l}0.320 \\
(0.020)\end{array}$ & $\begin{array}{l}0.157 \\
(0.003)\end{array}$ & $\begin{array}{l}0.216 \\
(0.001)\end{array}$ \\
\hline $\mathrm{C} 22: 1 \mathrm{n} 9$ & $\begin{array}{l}7.056 \\
(0.350)\end{array}$ & $\begin{array}{l}0.625 \\
(0.062)\end{array}$ & $\begin{array}{l}2.163 \\
(0.076)\end{array}$ & $\begin{array}{l}1.053 \\
(0.058)\end{array}$ & $\begin{array}{l}0.034 \\
(0.001)\end{array}$ & $\begin{array}{l}0.064 \\
(0.000)\end{array}$ & $\begin{array}{l}0.131 \\
(0.010)\end{array}$ & $\begin{array}{l}0.029 \\
(0.002)\end{array}$ & $\begin{array}{l}0.012 \\
(0.000)\end{array}$ & nd & $\begin{array}{l}0.035 \\
(0.001)\end{array}$ & $\begin{array}{l}0.038 \\
(0.000)\end{array}$ \\
\hline $\mathrm{C} 23: 0$ & $\begin{array}{l}2.125 \\
(0.089)\end{array}$ & $\begin{array}{l}0.112 \\
(0.003)\end{array}$ & $\begin{array}{l}0.474 \\
(0.024)\end{array}$ & $\begin{array}{l}0.049 \\
(0.000)\end{array}$ & $\begin{array}{l}0.301 \\
(0.031)\end{array}$ & $\begin{array}{l}0.197 \\
(0.017)\end{array}$ & $\begin{array}{l}0.022 \\
(0.002)\end{array}$ & $\begin{array}{l}0.109 \\
(0.005)\end{array}$ & $\begin{array}{l}0.036 \\
(0.002)\end{array}$ & $\begin{array}{l}0.061 \\
(0.001)\end{array}$ & $\begin{array}{l}0.188 \\
(0.010)\end{array}$ & $\begin{array}{l}0.060 \\
(0.001)\end{array}$ \\
\hline $\mathrm{C} 24: 0$ & $\begin{array}{l}2.913 \\
(0.120)\end{array}$ & $\begin{array}{l}0.284 \\
(0.024)\end{array}$ & $\begin{array}{l}0.889 \\
(0.004)\end{array}$ & $\begin{array}{l}0.182 \\
(0.000)\end{array}$ & $\begin{array}{l}2.409 \\
(0.080)\end{array}$ & $\begin{array}{l}1.774 \\
(0.055)\end{array}$ & $\begin{array}{l}0.120 \\
(0.004)\end{array}$ & $\begin{array}{l}0.238 \\
(0.012)\end{array}$ & $\begin{array}{l}0.105 \\
(0.003)\end{array}$ & $\begin{array}{l}0.498 \\
(0.002)\end{array}$ & $\begin{array}{l}0.975 \\
(0.040)\end{array}$ & $\begin{array}{l}0.342 \\
(0.012)\end{array}$ \\
\hline $\mathrm{C} 24: 1$ & $\begin{array}{l}8.428 \\
(0.371)\end{array}$ & $\begin{array}{l}1.229 \\
(0.043)\end{array}$ & $\begin{array}{l}1.957 \\
(0.086)\end{array}$ & $\begin{array}{l}2.747 \\
(0.150)\end{array}$ & $\begin{array}{l}0.145 \\
(0.002)\end{array}$ & $\begin{array}{l}0.242 \\
(0.017)\end{array}$ & $\begin{array}{l}0.234 \\
(0.009)\end{array}$ & $\begin{array}{l}0.021 \\
(0.001)\end{array}$ & $\begin{array}{l}0.028 \\
(0.001)\end{array}$ & nd & $\begin{array}{l}0.033 \\
(0.001)\end{array}$ & $\begin{array}{l}3.247 \\
(0.104)\end{array}$ \\
\hline Total & $\begin{array}{l}676.695 \\
(30.760)\end{array}$ & $\begin{array}{l}1770.906 \\
(51.528)\end{array}$ & $\begin{array}{l}1875.470 \\
(49.574)\end{array}$ & $\begin{array}{l}273.162 \\
(9.467)\end{array}$ & $\begin{array}{l}172.720 \\
(7.403)\end{array}$ & $\begin{array}{l}3175.035 \\
(0.099)\end{array}$ & $\begin{array}{l}30.526 \\
(1.500)\end{array}$ & $\begin{array}{l}460.109 \\
(0.035)\end{array}$ & $\begin{array}{l}35.026 \\
(2.248)\end{array}$ & $\begin{array}{l}830.621 \\
(43.528)\end{array}$ & $\begin{array}{l}1620.688 \\
(62.564)\end{array}$ & $\begin{array}{l}41.252 \\
(1.300)\end{array}$ \\
\hline SFA & $\begin{array}{l}325.294 \\
(15.000)\end{array}$ & $\begin{array}{l}18.138 \\
(1.251)\end{array}$ & $\begin{array}{l}24.794 \\
(1.978)\end{array}$ & $\begin{array}{l}31.348 \\
(1.234)\end{array}$ & $\begin{array}{l}80.791 \\
(5.348)\end{array}$ & $\begin{array}{l}30.345 \\
(2.356)\end{array}$ & $\begin{array}{l}9.930 \\
(0.740)\end{array}$ & $\begin{array}{l}12.691 \\
(1.101)\end{array}$ & $\begin{array}{l}7.801 \\
(0.433)\end{array}$ & $\begin{array}{l}26.953 \\
(10.464)\end{array}$ & $\begin{array}{l}24.732 \\
(15.735)\end{array}$ & $\begin{array}{l}16.305 \\
(0.938)\end{array}$ \\
\hline MUFA & $\begin{array}{l}222.648 \\
(12.345)\end{array}$ & $\begin{array}{l}1100.903 \\
(31.830)\end{array}$ & $\begin{array}{l}1187.203 \\
(32.674)\end{array}$ & $\begin{array}{l}163.922 \\
(6.535)\end{array}$ & $\begin{array}{l}71.945 \\
(5.240)\end{array}$ & $\begin{array}{l}2167.730 \\
(48.894)\end{array}$ & $\begin{array}{l}12.756 \\
(0.987)\end{array}$ & $\begin{array}{l}244.367 \\
(17.385)\end{array}$ & $\begin{array}{l}8.577 \\
(0.655)\end{array}$ & $\begin{array}{l}148.493 \\
(10.294)\end{array}$ & $\begin{array}{l}1284.685 \\
(40.547)\end{array}$ & $\begin{array}{l}16.985 \\
(0.861)\end{array}$ \\
\hline PUFA & $\begin{array}{l}128.753 \\
(7.435)\end{array}$ & $\begin{array}{l}651.864 \\
(23.000)\end{array}$ & $\begin{array}{l}663.473 \\
(24.332)\end{array}$ & $\begin{array}{l}77.892 \\
(2.546)\end{array}$ & $\begin{array}{l}19.984 \\
(1.201)\end{array}$ & $\begin{array}{l}976.961 \\
(30.400)\end{array}$ & $\begin{array}{l}7.840 \\
(0.649)\end{array}$ & $\begin{array}{l}203.051 \\
(15.385)\end{array}$ & $\begin{array}{l}18.648 \\
(1.200)\end{array}$ & $\begin{array}{l}655.176 \\
(34.929)\end{array}$ & $\begin{array}{l}311.271 \\
(25.755)\end{array}$ & $\begin{array}{l}7.962 \\
(0.500)\end{array}$ \\
\hline
\end{tabular}

a Results are expressed as mean (standard deviation) of three determinations. (C6:0) caproic acid; (C8:0) caprylic acid; (C10:0) capric acid; (C11:0) undecanoic acid; (C12:0) lauric acid; (C13:0) tridecanoic acid; (C14:0) myristic acid; (C14:1) myristoleic acid; (C15:0) pentadecanoic acid; (C16) palmitic acid; (C16:1) palmitoleic acid; (C17:0) heptadecanoic acid; (C17:1) cis-10-heptadecenoic acid; (C18:0) stearic acid; (C18:1) oleic acid; (C18:2) linoleic acid; (C18:3n6) $\gamma$-linolenic acid; (C18:3n3 + C20:1) $\alpha$-linolenic acid + cis-11-eicosaenoic acid; (C20:0) arachidic acid; (C20:2) cis-11,14-eicosadienoic acid; (C20:3n6) cis-8,11,14-eicosatrienoic acid (C20:4n6) arachidonic acid; (C20:5n3) cis-5,8,11,14,17-eicosapentaenoic acid; (C21:0) heneicosanoic acid; (C22:0) behenic acid; (C22:1 n9) erucic acid; (C23:0) tricosanoic acid; (C24:0) lignoceric acid; (C24:1) nervonic acid; (SFA) Saturated Fatty Acids; (MUFA) Monounsaturated Fatty Acids; (PUFA) Polyunsaturated Fatty Acids. nd - not detected. 


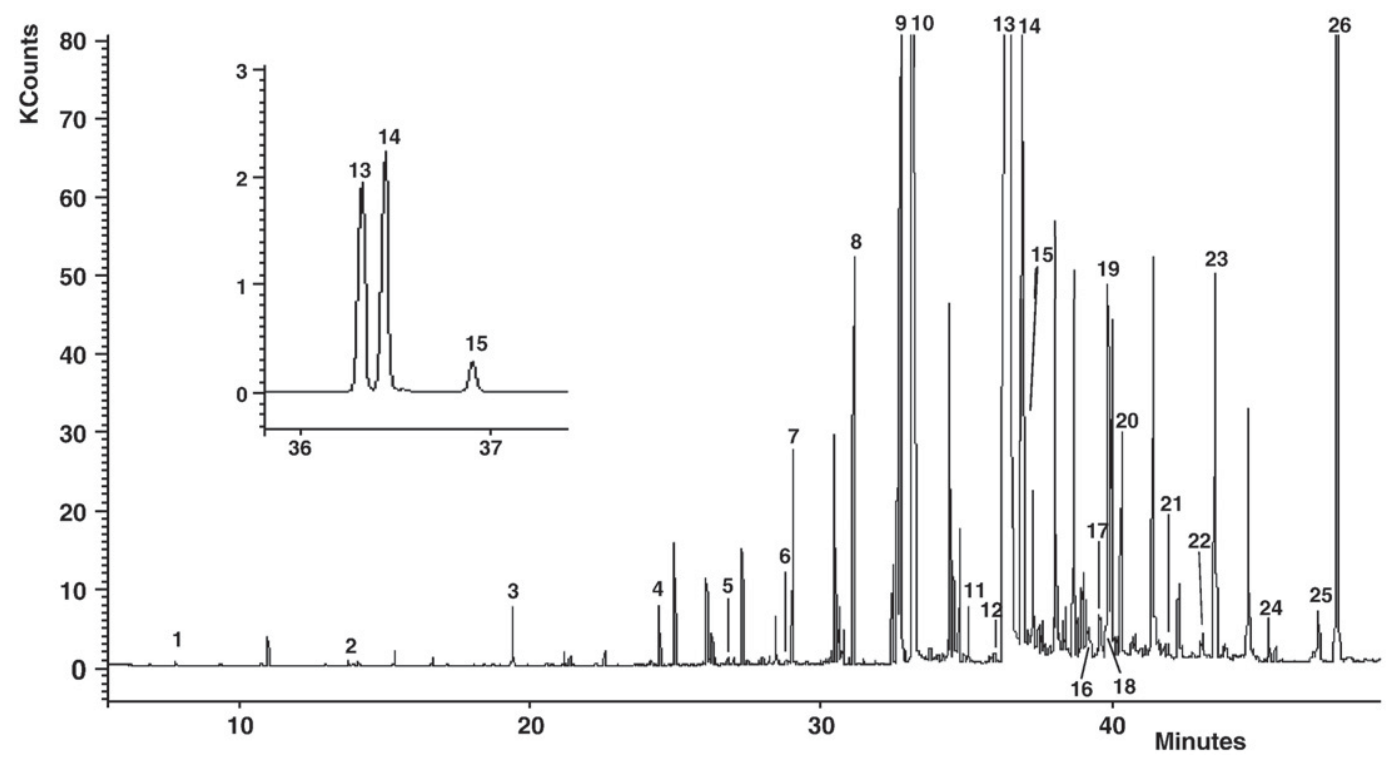

Fig. 1. GC-MS profile of methyl esters of free fatty acids of R. cyanoxantha mushroom. (1) caproic acid; (2) caprylic acid; (3) capric acid; (4) lauric acid; (5) tridecanoic acid; (6) myristoleic acid; (7) myristic acid; (8) pentadecanoic acid; (9) palmitoleic acid; (10) palmitic acid; (11) heptadecanoic acid; (12) $\gamma$-linolenic acid; (13) linoleic acid; (14) oleic acid; (15) stearic acid; (16) arachidonic acid; (17) cis-5,8,11,14,17-eicosapentaenoic acid; (18) cis-11,14-eicosadienoic; (19) cis-11-eicosenoic acid and $\alpha$-linolenic acid; (20) arachidic acid; (21) heneicosanoic acid; (22) erucic acid; (23) behenic acid; (24) tricosanoic acid; (25) nervonic acid; (26) lignoceric; (IS) Internal Standard. Chromatogram in full scan acquisition.

S. granulatus, S. luteus and $A$. caesarea revealed to be the richest species in what concerns to fatty acids amounts. H. rufescens, F. hepatica and B. edulis were the poorest ones, with the last containing the lowest contents (Table 2). In previous works, considering other edible mushrooms, the total fatty acids amounts found were higher than those observed for the present species [8,22].

The analysis of the obtained profiles showed that oleic (ca. 18 to $79 \%$ ), linoleic (ca. 11 to $79 \%$ ) and, to a lesser extent, palmitic (ca. 0.4 to $24 \%$ ) and stearic acids (ca. 0.2 to $16 \%$ of total compounds) were the main fatty acids in the studied species (Table 2). This is in agreement with the results reported for other edible mushrooms $[4,8,23]$. Oleic acid was the preponderant fatty acid in B. edulis, A. rubescens, $H$. agathosmus, T. equestre, S. bellini, H. rufescens, A. caesarea, S. granulatus, S. luteus and $R$. cyanoxantha species, while linoleic acid was the principal component in C. cibarius and F. hepatica species. H. rufescens and A. rubescens were the mushroom with the highest amounts of palmitic and stearic acids, respectively (Table 2 ).

Oleic acid (C18:1n9) is a monounsaturated fatty acid included in the omega- 9 family. Humans generally possess all the enzymes required for the synthesis of these metabolites, which means that oleic acid is not essential. Under severe conditions of essential fatty acids deprivation, mammals elongate and desaturate oleic acid to produce mead acid (C20:3n9) [7]. Oleic acid is found in olive oil and is known for its effectiveness in reducing cholesterol levels [24,25], which promotes the decrease of cardiovascular diseases [26]. Other omega-9 fatty acids, such as erucic, nervonic and cis-11-eicosenoic acids were also identified in the analyzed mushrooms (Table 2).

Linoleic acid (LA) is an essential fatty acid as it cannot be synthesised by the human organism, due to the lack of desaturase enzymes required for its production. It must be obtained from the diet and originates the omega- 6 fatty acids series, which includes $\gamma$-linolenic (GLA), dihomo- $\gamma$-linolenic (DGLA) and arachidonic (AA) acids [7]. It is known that linoleic acid is the precursor of eightcarbon volatiles in fungi, such as 1-octen-3-ol, 3-octanol, 1-octen-3-one and 3-octanone [27], which are the principal aromatic compounds in most species [28], contributing also to the flavour of most of the mushroom species analysed in the present study [1].

$\alpha$-Linolenic acid (ALA) is an essential fatty acid able to produce the omega-3 fatty acids series in humans, including eicosapentaenoic acid (EPA) and docosahexaenoic acid (DHA) [7].
The omega- 3 and -6 fatty acids are the biosynthetic precursors of eicosanoids, meaning that their intake concentrations will strongly influence eicosanoids production, and, therefore, the organism's metabolic functions $[7,29]$. Beyond these, many works showed that they can also decrease the total amount of fat in blood (cholesterol), reducing the risk of cardiovascular diseases [7,30-32]. A deficient intake of EFA (Essential Fatty Acids) can be responsible for many problems, such as dermatitis, imunosupression and cardiac disfunctions [5]. In addition to linoleic and $\alpha$-linolenic acids, in our study some of the essential metabolites belonging to omega- 6 and omega- 3 series were identified in some of the analysed species (Table 2). GLA was found in S. bellini, S. luteus, H. agathosmus, C. cibarius, $R$. cyanoxantha, B. edulis and $F$. hepatica species (ca. 9 to $1532 \mu \mathrm{g} / \mathrm{kg}$ ), GLA was observed in $R$. cyanoxantha and T. equestre species (ca. 19 to $22 \mu \mathrm{g} / \mathrm{kg}$ ) and EPA was detected in $H$. rufescens, $R$. cyanoxantha, A. rubescens and F. hepatica (16 to $44 \mu \mathrm{g} / \mathrm{kg}$ ) (Table 2).

Since humans are limited in the obtainment of EPA from linolenic acid, they need a supplementary diet intake to maintain the recommended levels of this acid in the organism [33]. The results of the present work revealed that EPA is not present in almost all of the analysed species and that the mushroom species in which it is detected exhibit very low amounts of this compound (Table 2). The minor contents of EPA were already reported by other authors in other mushroom species [34]. DHA was also studied by us but it was not detected in any of the analysed mushroom species (data not shown). In opposition to other matrices, like oily fish and fish oil [31], mushrooms would not be a good diet source of DHA. This is the most abundant omega-3 PUFA contained in the tissues, and it is especially necessary for optimal visual acuity and neural development [35]. In fact, by the observation of Table 2, omega- 6 (except linoleic acid), as well as omega-3 fatty acids appeared in the studied mushroom species in very low amounts. Although these compounds are not abundant, they are essential for some important human metabolic pathways [6].

The characterization of saturated, monounsaturated and polyunsaturated fatty acid profiles is shown in Table 2 and Fig. 2. The total fatty acid contents for SFA, MUFA and PUFA ranged from ca. 8 to $325 \mathrm{mg} / \mathrm{kg}$, ca. 9 to $2168 \mathrm{mg} / \mathrm{kg}$ and ca. 8 to $977 \mathrm{mg} / \mathrm{kg}$, respectively. SFA content was higher in A. rubescens due to palmitic acid (47\%) and in S. bellini as result of the high levels of both palmitic and stearic acids 


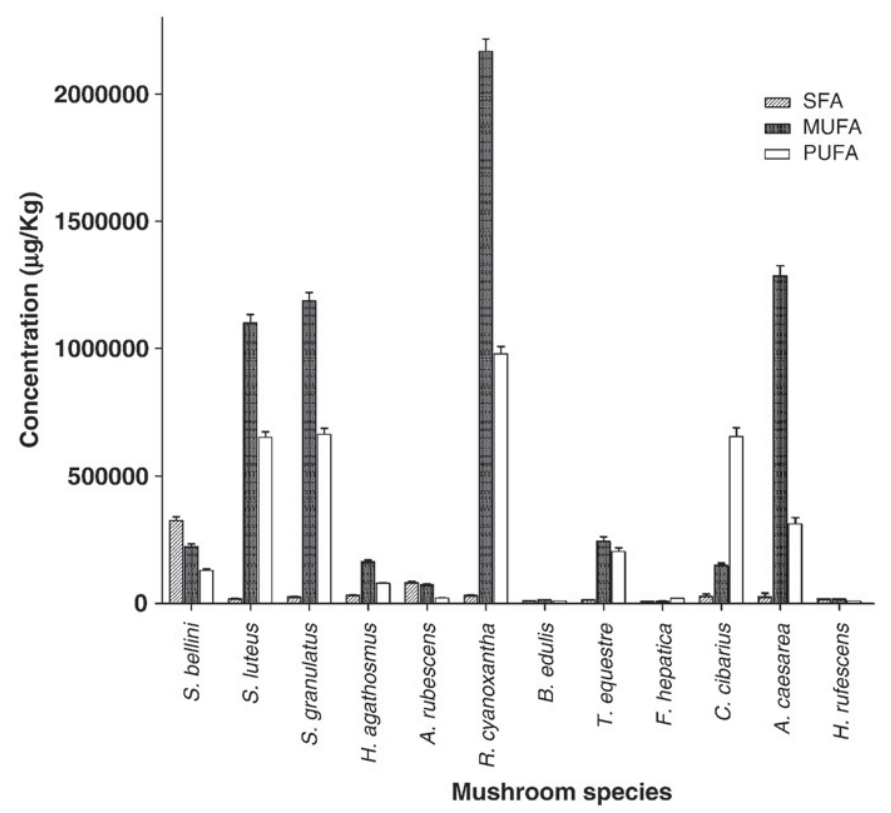

Fig. 2. SFA (Saturated Fatty Acids), MUFA (Monounsaturated Fatty Acids) and PUFA (Polyunsaturated Fatty Acids) contents in wild edible mushrooms species: Suillus bellini, Suillus luteus, Suillus granulatus, Hygrophorus agathosmus, Amanita rubescens, Russula cyanoxantha, Boletus edulis, Tricholoma equestre, Fistulina hepatica, Cantharellus cibarius, Amanita caesarea and Hydnum rufescens. Results are expressed as $\mu \mathrm{g} / \mathrm{kg}$ dry matter. Values show mean \pm SE from 3 experiments performed in triplicate.

( $48 \%$ of total compounds) (Table 2). PUFAs and MUFAs were predominant in all of the remaining mushroom species, varying from ca. 12 to $79 \%$ and from 18 to $79 \%$ of total fatty acids, respectively (Table 2 ). The high linoleic acid content contributes to the overall increase in PUFAs, while oleic acid raises MUFAs amounts. PUFAs amount was higher in $C$. cibarius and $F$. hepatica species, in which linoleic acid was the main compound (ca.79 and 53\% of total fatty acids, respectively), and MUFA were present in highest levels in S. luteus, S. granulatus, $H$. agathosmus, $R$. cyanoxantha, $B$. edulis, T. equestre, A. caesarea and H. rufescens species, due to oleic acid (ca. 29 to 79\%). This is consistent with the predominance of unsaturated fatty acids over the saturated ones in mushrooms total fatty acid content previously observed $[23,34,36]$. The substitution of saturated by unsaturated fatty acids in the diet is very important, as it leads to an increase in HDL cholesterol and decreases LDL cholesterol and triacylglycerols [37]. On the other hand, it was proved that a diet rich in saturated fat is correlated with an increase in atherosclerosis and coronary heart disease [38]. Based on these findings, the consumption of $A$. rubescens and S. bellini should not be so advantageous as the intake of the remaining species.

The fatty acid profiles of the different mushroom species appeared to be distinct. Although oleic and linoleic acids were present in all of them as the most abundant compounds, linoleic acid was the major compound in $C$. cibarius and $F$. hepatica species, while oleic acid was the main one in the remaining species (Table 2). Nevertheless, it seems that minor fatty acids, such as omega-3 and -6 (except linoleic acid) could be the main responsible for the distinct profiles of the analyzed mushroom species. Based in Table 2, we can see that the presence of these fatty acids occurs only in certain species and that there are great quantitative differences on these compounds between the mushroom species. Maybe the genes responsible for the synthesis of the enzymes participating in the biosynthetic pathways for the formation of these fatty acids could be distinct [39].

For a deeper comparison of the samples, we performed an Agglomerative Hierarchic Cluster Analysis (HCA) regarding mushroom species and fatty acids variables. The fatty acids profiles allowed to separate the mushrooms species into 5 groups: group 1 included only S. bellini; group 2 comprised only A. rubescens; group 3 contained
H. agathosmus, C. cibarius, A. caesarea and R. cyanoxantha; group 4 was composed by B. edulis, T. equestre and $F$. hepatica and group 5 consisted of H. rufescens, S. granulatus and S. luteus (Fig. 3).

Group 2 is distinct from group 1: undecanoic, arachidonic and caprylic acids were not detected in $A$. rubescens but were present in $S$. bellini. On the contrary, $\alpha$-linolenic and cis-5,8,11,14,17-eicosapentaenoic acids were found in A. rubescens, but not in S. bellini (Table 2). Groups 3 and 5 are the unique containing myristoleic acid (Table 2). Additionally, all of the members of group 5 exhibit cis-10-heptadecenoic acid. Group 4 is characterized by the presence of capric, arachidic and heneicosanoic acids and by the absence of myristoleic acid in all of their species (Table 2).

The determination of the total fatty acids profile of $S$. luteus showed, besides the 14 already reported [1,40], additional 14 compounds described for the first time (Table 2). As far as we know, $\gamma$-linolenic acid was the only reported fatty acid [40] that was not identified in the present work. The results from our study (Table 2) and others [40] allowed to notice that oleic and linoleic acids are the major fatty acids in S. luteus.

The fatty acids analysis of $S$. granulatus revealed 20 compounds in common with the literature [1,3,41] plus 6 more compounds found for the first time: undecanoic, myristoleic, cis-10-heptadecenoic, cis8,11,14-eicosatrienoic, heneicosanoic and tricosanoic acids (Table 2). Caproic acid, which was already reported $[1,3]$ in this species, was not found now. The present study identified linoleic acid, followed by oleic acid as the major compounds (Table 2), which is in good agreement with previous works $[3,41]$.

For S. bellini, only palmitoleic acid was reported until now [1], but other 22 fatty acids were now identified. The main fatty acids found followed the order oleic $>$ palmitic $>$ linoleic $>$ stearic acid (Table 2).

The comparison of the results obtained with the three analysed Suillus species (S. luteus, S. granulatus and S. bellini) allows to observe that $S$. granulatus and S. luteus presented higher fatty acid contents

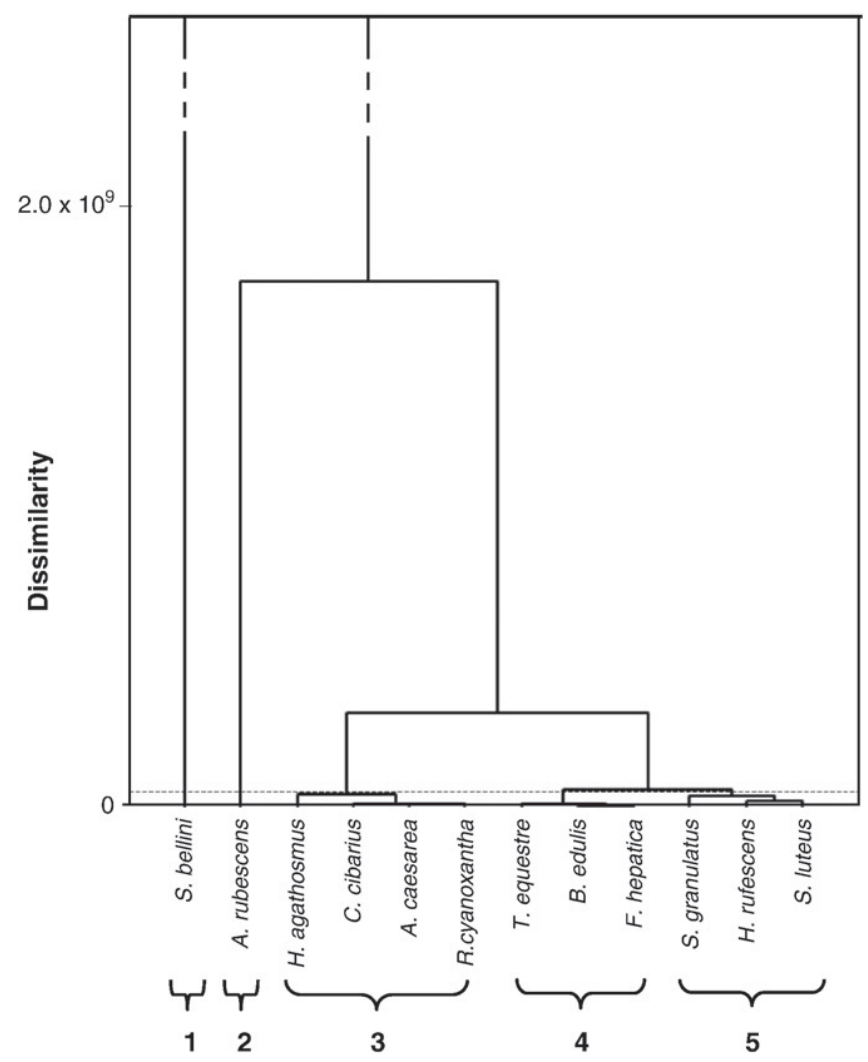

Fig. 3. Dendogram of edible mushroom species with fatty acids contents. 1-5 correspond to different grouping species. 
than S. bellini (Table 2). In what concerns to their qualitative profiles, the species revealed to be distinct. Despite the 23 fatty acids common to the three species, caproic acid was identified only in S. luteus; arachidonic acid was not found in S. granulatus and S. bellini lacked myristoleic, cis-10-heptadecenoic, $\alpha$-linolenic and cis-11-eicosenoic acids (Table 2). Concerning their quantitative profiles, both S. granulatus and S. luteus exhibited oleic and linoleic acids as the major compounds (ca. 63 and 35\% of total fatty acids for S. granulatus and ca. 62 and 37\% for S. luteus, respectively) and all the other fatty acids in trace amounts (Table 2 ). The presence of four main fatty acids in S. bellini, following the order oleic $>$ palmitic $>$ linoleic $>$ stearic acids, distinguishes this from the other Suillus species and contributes for gathering $S$. granulatus and S. luteus in a group and S. bellini in a different one (Fig. 3). This differentiation was also found before [1].

As for $R$. cyanoxantha, in addition to the fatty acids previously described in the literature, namely caproic, palmitoleic, stearic and oleic acids [1], other 23 compounds were identified for the first time (Table 2, Fig. 1). Oleic and linoleic acids were the preponderant compounds accounting for ca. $68 \%$ and $31 \%$ of total fatty acids, respectively (Table 2 ).

Concerning A. rubescens, our work revealed lauric, tridecanoic, cis5,8,11, 14,17-eicosapentaenoic, cis-8,11,14-eicosatrienoic, cis-11,14eicosadienoic, cis-11-eicosenoic, $\alpha$-linolenic, heneicosanoic, erucic, tricosanoic, nervonic and lignoceric acids for the first time, apart from other 11 fatty acids described before $[1,8,40]$ (Table 2 ). As reported in the literature, the obtained results showed that the major compounds were oleic, palmitic [8], stearic [1] and [8] and linoleic acids [8,40]. Both qualitative and quantitative fatty acid profiles of $A$. rubescens were very different from those of $A$. caesarea, placing these Amanita species in two distinct groups (Fig. 3).

In what concerns to $H$. agathosmus, 24 fatty acids were now found in addition to caproic, palmitoleic and oleic acids previously described [1] (Table 2). Oleic acid revealed to be the main compound in H. agathosmus (ca. 58\% of total compounds), followed by linoleic acid (ca. $28 \%$ of total compounds) (Table 2 ).

With respect to $B$. edulis, our work revealed 4 additional fatty acids not previously found in the literature: cis-10-heptadecenoic, arachidonic, cis-8,11,14-eicosatrienoic and heneicosanoic acids (Table 2). The remaining fatty acids identified in our work were already described $[1,3,9,23,36]$. Caproic, caprylic, capric $[3,9]$, undecanoic and tridecanoic acids [3] found in other studies, were not detected. By observing Table 2 , it is possible to identify oleic acid, followed by linoleic, palmitic and stearic acids as the main fatty acids of $B$. edulis. Previous studies also classified oleic and linoleic acids as the most abundant fatty acids in this species, but, in opposition to our work, linoleic acid was present in highest amounts than oleic acid. Palmitic and stearic acids identified in the preceding studies were also prevalent fatty acids, but to a lesser extent $[3,9,23,36]$.

As far as we are aware, this is the first work describing the presence of 23 fatty acids in F. hepatica (Table 2), 7 of which were already reported in the literature $[1,10,42]$. The main fatty acids found now were linoleic $>$ oleic $>$ palmitic acid (Table 2).

As far as we know, this is the first report to describe the presence of 20 fatty acids in T. equestre (Table 2): in addition to those already reported (caproic, palmitoleic and stearic acids) [1], 17 more fatty acids were found in this species. The results of the present work showed that oleic and linoleic represent the major fatty acids of T. equestre, corresponding to ca. $53 \%$ and $44 \%$ of total compounds, respectively (Table 2 ).

The determination of the total fatty acids profile of $C$. cibarius revealed 15 fatty acids in common with the literature (Table 2) $[9,34,36]$ and arachidonic acid, which was identified for the first time in this species. On the other hand, compounds such as myristoleic [36] and other fatty acids $[9,34]$ were not found in our work.

Concerning the species described above, their qualitative and quantitative fatty acids profiles revealed, to some extent, to be different from those described in the literature. This could be explained by the diversity of extraction, derivatization or quantification methods used in the different studies. In addition, the different geographical origin and/ or stage of development of the analysed species also cannot be excluded.

\section{Conclusion}

In conclusion, the chemical composition of Portuguese wild edible mushrooms clearly indicates that they provide key nutrients, such as unsaturated fatty acids. As far as we know, the fatty acid profiles of A. caesarea and $H$. rufescens were described for the first time. For the remaining species, a high number of new compounds were now reported, which improved the knowledge about their fatty acids profiles. The studied species proved to be an excellent food, which can be used in low caloric diets, due to their low saturated fat contents, and, therefore, can be consumed by people with high blood cholesterol levels. Because of the diversity of unsaturated fatty acids in mushrooms, which is of great importance to the metabolic pathways and to human health, this food could also be used to make nutritive supplements by food industry.

\section{Acknowledgement}

Bárbara Ribeiro is indebted to Fundação para a Ciência e a Tecnologia (FCT) for a grant (SFRH/BD/22108/2005).

\section{References}

[1] P. Guedes de Pinho, B. Ribeiro, R.F. Gonçalves, P. Baptista, P. Valentão, R.M. Seabra, P.B Andrade, Correlation between the pattern volatiles and the overall aroma of wild edible mushrooms, J. Agric. Food Chem. 56 (2008) 1704-1712.

[2] P. Mattila, K. Suonpaa, V. Piironen, Functional properties of edible mushrooms, Nutrition 16 (2000) 694-696.

[3] K. Pedneault, P. Angers, A. Gosselin, R.J. Tweddell, Fatty acid composition of lipids belonging to the family Boletaceae, Mycol. Res. 110 (2006) 1179-1183.

[4] L. Barros, P. Baptista, D.M. Correia, J.S. Morais, I.C.F.R. Ferreira, Effects of conservation treatment and cooking on the chemical composition and antioxidant activity of Portuguese wild edible mushrooms, J. Agric. Food Chem. 55 (2007) 4781-4788.

[5] C.A. Burtis, E.R. Ashwood, Tietz Fundamentals of Clinical chemistry, 4th ed. W. B. Saunders Company, Philadelphia, 1996.

[6] M.J. Gibney, H.H. Vorster, F.J. Kok, Introduction to Human Nutrition, Blackwell Science, Oxford, 2002.

[7] D. Voet, J.G. Voet, Biochemistry, 3rd ed. Wiley \& Sons, Hoboken, N. J., 2004.

[8] M.F. León-Guzmán, I. Silva, M.G. López, Proximate chemical composition, free amino acid contents, and free fatty acid contents of some wild edible mushrooms from Querétaro, México, J. Agric. Food Chem. 45 (1997) 4329-4332.

[9] L. Barros, T. Cruz, P. Baptista, L.M. Estevinho, I.C.F.R. Ferreira, Wild and commercial mushrooms as source of nutrients and nutraceuticals, Food Chem. Toxicol. 46 (2008) 2742-2747.

[10] S. Wu, H. Zorn, U. Krings, R.G. Berger, Volatiles from submerged and surfacecultured beefsteak fungus, Fistulina hepatica, Flavour Frag. J. 22 (2007) 53-60.

[11] Library 4 Science (2008) Gas Chromatography by RPW Scott part of the Chrom Ed. Series. Retrieved March 26, 2008 from www.chromatography-online.org/GC/ Quantitative-Analysis/Derivatization/rs56.html.

[12] P. Valentão, P.B. Andrade, J. Rangel, B. Ribeiro, B. Silva, P. Baptista, R.M. Seabra, Effect of the conservation procedure on the contents of phenolic compounds and organic acids in chanterelle (Cantharellus cibarius) mushroom, J. Agric. Food Chem. 53 (2005) 4925-4931.

[13] B. Ribeiro, P. Valentão, P. Baptista, R.M. Seabra, P.B. Andrade, Phenolic compounds and organic acids contents and antioxidative properties of beefsteak fungus (Fistulina hepatica), Food Chem. Toxicol. 45 (2007) 1805-1813.

[14] B. Ribeiro, J. Rangel, P. Valentão, P. Baptista, R.M. Seabra, P.B. Andrade, Contents of carboxylic acids and two phenolics and antioxidant activity of dried Portuguese wild edible mushrooms, J. Agric. Food Chem. 54 (2006) 8530-8537.

[15] B. Ribeiro, R. Lopes, P.B. Andrade, R.M. Seabra, R.F. Gonçalves, P. Baptista, I. Quelhas, P. Valentão, Comparative study of phytochemicals and antioxidant potential of wild edible mushroom caps and stipes, Food Chem. 110 (2008) 47-56.

[16] C. Bas, T.W. Kuyper, M.E. Noordeloos, E.C. Vellinga, Flora Agaricina Neerlandica: Critical monographs on families of agarics and boleti occuring in the Netherlands, Volume 1-5, A. A Balkema, Rotterdam, 1990-2001.

[17] M. Bon, Guia de campo de los hongos de Europa, Ediciones Omega, Barcelona, 1988

[18] R. Courtecuisse, Mushrooms of Britain and Europe, HarperCollins Publishers, London, 1999.

[19] R. Courtecuisse, B. Duhem, Mushrooms and toadstools of Britain and Europe, HarperCollins Publishers, London, 1995.

[20] A. Marchand, Champignons du Nord et du Midi, Tome 1-9, Soc. Mycol. Pyrénées Mediterranéenes, Perpignan, 1971-1986. 
[21] M. Moser, Keys to Agarics and Boleti (Poliporales, Boletales, Agaricales, Russulales), Roger Phillips, London, 1983.

[22] Y. Hadar, E. Cohen-Arazi, Chemical composition of the edible mushroom Pleurotus ostreatus produced by fermentation, Appl. Environ. Microb. (1986) 1352-1354 June.

[23] N. Yilmaz, M. Solmaz, I. Türkedul, M. Elmastaş, Fatty acid composition in some wild edible mushrooms growing in the middle Black Sea region of Turkey, Food Chem. 99 (2006) 168-174.

[24] C. Puiggrós, P. Chacón, L.I. Armadans, J. Clapés, M. Planas, Effects of oleic-rich and omega-3-rich diets on serum lipid pattern and lipid oxidation in mildly hypercholesterolemic patients, Clin. Nutr. 21 (2002) 79-87.

[25] M. Tomás, M. Sentí, R. Elosua, J. Vila, J. Sala, R. Masià, J. Marrugat, Interaction between the Gln-Arg 192 variants of the paraoxonase gene and oleic acid intake as a determinant of high-density lipoprotein cholesterol and paraoxonase activity, Eur. J. Pharmacol. 432 (2001) 121-128.

[26] Y.M. Pacheco, S. López, B. Bermúdez, R. Abia, J. Villar, F.J.G. Muriana, A meal rich in oleic acid beneficially modulates postprandial sICAM-1 and SVCAM-1 in normotensive and hypertensive hypertriglyceridemic subjects, J. Nutr. Biochem. 19 (2008) 200-205.

[27] E. Combet, J. Henderson, D.C. Eastwood, K.S. Burton, Eight-carbon volatiles in mushrooms and fungi: properties, analysis, and biosynthesis, Mycoscience 47 (2006) 317-326.

[28] J. Maga, Mushroom flavor, J. Agric. Food Chem. 29 (1981) 1-4.

[29] E.C. Borsonelo, J.C.F. Galduróz The role of polyunsaturated fatty acids (PUFAs) in development, aging and substance abuse disorders: review and propositions, Prostaglandin Leukot. Essent. 78 (2008) 237-245.

[30] J.M. Geleijnse, E.J. Giltay, D.E. Grobbee, A.R.T. Donders, F.J. Kolk, Blood pressure response to fish oil supplementation: metaregression analysis of randomized trials, J. Hypertens. 20 (2002) 1493-1499.
[31] P.M. Kris-Etherton, W.S. Harris, L. Appel, Fish consumption, fish oil, omega-3 fatty acids, and cardiovascular disease, Circulation 106 (2002) 2747-2757.

[32] W.E. Connor, Importance of n-3 fatty acids in health and disease, Am. J. Clin. Nutr. 71 (2000) 171S-175S

[33] E.M. Balk, A.H. Lichtenstein, M. Chung, B. Kupelnick, P. Chew, J. Lau, Effects of omega-3 fatty acids on serum markers of cardiovascular disease risk: a systematic review, Atherosclerosis 189 (2006) 19-30.

[34] L. Barros, B.A. Venturini, P. Baptista, L.M. Estevinho, I.C.F.R. Ferreira, Chemical composition and biological properties of Portuguese wild mushrooms: a comprehensive study, J. Agric. Food Chem. 56 (2008) 3856-3862.

[35] A.A. Spector, Essentiality of fatty acids, Lipids 34 (1999) S1-S3.

[36] S. Kavishree, J. Hemavathy, B.R. Lokesh, M.N. Shashirekha, S. Rajarathnam, Fat and fatty acids of Indian edible mushrooms, Food Chem. 106 (2008) 597-602.

[37] J. Isanga, G.N. Zhang, Biologically active components and nutraceuticals in peanuts and related products: review, Food Rev. Int. 23 (2007) 123-140.

[38] L. Wang, A.R. Folsom, J.H. Eckfeldt, Plasma fatty acid composition and incidence of coronary heart disease in middle aged adults: the Atherosclerosis Risk in Communities (ARIC) Study, Nutr. Metab. Cardiovas. 13 (2003) 256-266.

[39] B. Qi, T. Fraser, S. Mugford, G. Dobson, O. Sayanova, J. Butler, J.A. Napier, A.K. Stobart, C.M. Lazarus, Production of very long chain polyunsaturated omega-3 and omega-6 fatty acids in plants, Nat. Biotechnol. 22 (2004) 739-745.

[40] L. Karliński, B. Ravnskov, B. Kieliszewska-Rokicka, J. Larsen, Fatty acid composition of various ectomycorrhhizal fungi and ectomycorrhizas of Norway spruce, Soil Biol. Biochem. 39 (2007) 854-866.

[41] J.L. Sumner, The fatty acid composition of basidiomycetes, New Zeal. J. Bot. 11 (1973) 435-442.

[42] S. Wu, U. Krings, H. Zorn, R.G. Berger, Volatile compounds from the fruiting bodies of beefsteak fungus Fistulina hepatica (Schaeffer: Fr.) Fr, Food Chem. 92 (2005) 221-226. 